\title{
ГЕНДЕРНЫЕ ОСОБЕННОСТИ АССОЦИАЦИЙ ПОЛИМОРФИЗМА ГЕНОВ МАТРИКСНЫХ МЕТАЛЛОПРОТЕИНАЗ С РАЗВИТИЕМ ЯЗВЕННОЙ БОЛЕЗНИ У НАСЕЛЕНИЯ ЦЕНТРАЛЬНОГО ЧЕРНОЗЕМЬЯ РОССИИ
}

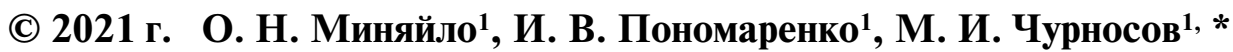 \\ ${ }^{1}$ Белгородский государственный национальный исследовательский университет, Белгород, 308015 Россия \\ *e-mail: churnosov@bsu.edu.ru \\ Поступила в редакцию 17.12.2020 г. \\ После доработки 23.01.2021 г. \\ Принята к публикации 11.02.2021 г.
}

\begin{abstract}
Изучены гендерные особенности ассоциаций функционально значимых полиморфных локусов генов матриксных металлопротеиназ с развитием язвенной болезни (ЯБ) у населения европейской части России. В исследование включены 305 мужчин (больные ЯБ - 188, контрольная группа - 117) и 441 женщина (больные ЯБ - 211, контрольная группа - 230). Проведено генотипирование десяти функционально значимых полиморфных локусов генов матриксных металлопротеиназ: rs 1799750 MMP1, rs243865 MMP2, rs679620 MMP3, rs1940475 MMP8, rs3918242, rs3918249, rs17576, rs3787268, rs2250889, rs 17577 гена ММР9. Ассоциации полиморфных локусов генов ММР с ЯБ изучались методом логистической регрессии (оценивались доминантная, рецессивная и аддитивная генетические модели взаимодействия аллелей). Установлено, что факторами риска возникновения ЯБ у мужчин были аллели $C \operatorname{rs} 3918249\left(\mathrm{OШ}=1.61, p_{\text {perm }}=0.048\right)$ и $G \operatorname{rs} 17576$ (ОШ $\left.=1.48-2.08, p_{\text {perm }} \leq 0.042\right)$ гена $M M P 9$. У женщин протективное значение для развития заболевания имел аллель $2 G$ rs 1799750 гена $M M P 1\left(\mathrm{Ш}=0.74, p_{\text {perm }}=0.047\right)$. Полиморфные локусы, ассоциированные с ЯБ у мужчин и женщин, проявляют выраженные эпигенетические эффекты (влияют на афинность мотивов ДНК к различным транскрипционным факторам, расположены в регионе промоторов и энхансеров в желудке и двенадцатиперстной кишке, связаны с экспрессией генов в различных отделах пищеварительной системы). Выявлены гендерные особенности ассоциаций полиморфизма генов $M M P$ с развитием ЯБ: rs3918249 и rs17576 гена ММР9 определяют подверженность к развитию ЯБ у мужчин, а rs1799750 гена $M M P 1-$ у женщин.
\end{abstract}

Ключевые слова: язвенная болезнь, матриксные металлопротеиназы, полиморфизм, ассоциации, пол.

DOI: $10.31857 / \mathrm{S} 0016675821100088$

Язвенная болезнь (ЯБ) желудка и двенадцатиперстной кишки продолжает оставаться одним из наиболее часто встречающихся заболеваний пищеварительной системы в различных странах миpa [1]. В Соединенных Штатах Америки ежегодно регистрируется до 500 тыс. новых случаев заболеваемости ЯБ [2]. Согласно официальным статистическим материалам Росстата за 2018 г. язвенная болезнь желудка и двенадцатиперстной кишки была зарегистрирована у 834.8 на 100000 жителей России. При этом в последние годы в РФ наблюдается тенденция некоторого снижения заболеваемости язвенной болезнью желудка и двенадцатиперстной кишки с 95.6 случаев с впервые установленным диагнозом на 100000 населения в 2010 г. до 71.9 на 100000 населения в 2018 г. [3]. Имеются гендерные особенности встречаемости ЯБ: считается, что среди пациентов с дуоденальными язвами преобладают мужчины, а среди больных с язвами желудка соотношение женщин и мужчин оказывается примерно одинаковым [4].

В соответствии с современными представлениями об этиопатогенезе ЯБ при развитии заболевания происходит нарушение в равновесии между факторами агрессии (кислотно-пептическое содержимое желудка) и защитными механизмами слизистой оболочки желудка и двенадцатиперстной кишки [4]. В настоящее время ключевое значение в развитии ЯБ отводится бактериям H. pylori [5]. Эти микроорганизмы продуцируют различные ферменты - уреазы, протеазы, фосфолипазы, повреждающие защитный барьер слизистой оболочки. Также $H$. pylori вырабатывает цитотоксины, индуцирующие высвобождение в слизистой оболочке желудка различных цитокинов (факторов некроза опухолей, интерлейкинов 
и др.), вызывающих развитие в ней воспалительного процесса [4, 5]. Согласно литературным данным c $H$. pylori ассоциировано около $80 \%$ язвенных поражений двенадцатиперстной кишки и 60\% язв желудка [6].

Следует отметить, что возникновение воспалительного процесса в слизистой оболочке желудка и двенадцатиперстной кишки обусловливает вовлечение в патогенез ЯБ матриксных металлопротеиназ (ММР), которые играют ключевую роль в деградации и ремоделировании внеклеточного матрикса во время процессов воспаления и ранозаживления, и в том числе при H. pylori инфекции $[7,8]$. В ряде работ показаны более высокие уровни матриксных металлопротеиназ (ММР3, ММР9 и др.) в месте локализации язвенного дефекта и их корреляция с уровнями IL $1 \beta$, IL6 и IL8, а также снижение содержания MMP при эффективном лечении ЯБ [9-13]. Важно подчеркнуть, что одну из ключевых ролей в индукции синтеза ММР играет $H$. pylori инфекция $[11,12]$. Продемонстрирована значимая связь полиморфизма генов $M M P$ с восприимчивостью к язвенной болезни [13, 14]. При этом следует отметить, что в Российской Федерации выполнена лишь одна работа по изучению ассоциаций полиморфизма генов матриксных металлопротеиназ с развитием ЯБ у жителей Республики Башкортостан [14], что является недостаточным и диктует необходимость проведения дальнейших молекулярно-генетических исследований ЯБ в различных этно-территориальных популяциях РФ и в том числе направленных на выявление гендерных особенностей вовлеченности полиморфизма генов $M M P$ в развитие ЯБ.

Цель настоящей работы - анализ гендерных особенностей ассоциаций полиморфных локусов генов матриксных металлопротеиназ с формированием язвенной болезни желудка и двенадцатиперстной кишки у населения Центрального Черноземья России.

\section{МАТЕРИАЛЫ И МЕТОДЫ}

Выборка для данного исследования включала 746 человек. Среди них мужчины составляли 305 человек (больные ЯБ - 188, контрольная группа 117), а женщины - 441 человек (больные ЯБ - 211, контрольная группа - 230). Среди женщин, больных ЯБ, 149 индивидуумов имели язвенную болезнь желудка (70.61\%) и 62 пациента - язвенную болезнь двенадцатиперстной кишки (29.39\%). Среди мужчин, больных ЯБ, данные показатели составили 68 (36.17\%) и 120 (63.83\%) человек соответственно. Выборка для исследования формировалась из индивидуумов русской национальности, родившихся и проживающих в Центральном Черноземье РФ $[15,16]$, не состоящих в родстве между собой. Пациенты включались в исследование только после установления диагноза, подтвержденного с помощью клинических и лабораторно-инструментальных методов обследования [1]. Диагностика язвенной болезни проводилась на базе отделения гастроэнтерологии Белгородской областной клинической больницы Святителя Иоасафа. Всем пациентам было выполнено эндоскопическое исследование желудка и двенадцатиперстной кишки (гастродуоденоскопия) с последующей биопсией. Для определения H. pylori проводилось морфологическое исследование биоптатов слизистой оболочки. H. pylori была выявлена у 202 больных ЯБ (50.62\% от всех больных), в том числе у 100 мужчин с ЯБ (53.19\% от всех мужчин, больных ЯБ) и у 102 женщин с ЯБ (48.34\% от всех женщин, больных ЯБ). В контрольную группу включались индивидуумы соответствующего группе больных пола, без клинических и эндоскопических признаков язвенной болезни, сопоставимые с больными по возрасту. Средний возраст изучаемой группы мужчин, больных ЯБ, и контрольной группы составил $48.24 \pm 13.63$ и $48.64 \pm 13.52$ лет соответственно $(p>0.05)$, среди женщин средний возраст был равен $49.04 \pm 12.53$ лет (больные ЯБ) и $49.23 \pm$ \pm 12.75 лет (контрольная группа) $(p>0.05)$. Исследование проводилось под контролем этического комитета медицинского института Белгородского государственного национального исследовательского университета с информированного согласия всех обследуемых индивидуумов.

Материалом для молекулярно-генетического исследования послужили образцы ДНК, выделенные из периферической венозной крови фенольно-хлороформным методом. Для исследования были отобраны десять полиморфных локусов генов матриксных металлопротеиназ: rs1799750 MMP1, rs243865 MMP2, rs679620 MMP3, rs1940475 MMP8, rs3918242, rs3918249, rs17576, rs3787268, rs2250889, rs17577 гена ММР9. Включенные в исследование локусы являются регуляторными полиморфными вариантами (rSNP) [17, 18], согласно базе данных HaploReg (v4.1.) (http://archive.broadinstitute.org/mammals/haploreg/haploreg.php) они имеют значимый регуляторный потенциал. Амплификацию полиморфных локусов проводили с помощью полимеразной цепной реакции на амплификаторе CFX96 (Bio-Rad) методом TaqMan зондов (использовались наборы, синтезированные ООО “Тест-Ген” (Ульяновск)).

Ассоциации полиморфных локусов генов MМР с ЯБ изучались методом логистической регрессии (оценивались доминантная, рецессивная и аддитивная генетические модели взаимодействия аллелей) с использованием программы PLINK v 2.050 (http://pngu.mgh.harvard.edu/Èpurcell/plink). Характер ассоциаций оценивался на основе показателя отношения шансов (ОШ) и его 95\%-ного доверительного интервала (95\% ОШ). Коррекцию на множественные сравнения проводили с использованием адаптивного пермутацион- 
ного теста $\left(p_{\text {perm }}\right)$. Статистически значимым считали уровень $p_{\text {perm }}<0.05$.

Для полиморфных локусов генов $M M P$, показавших значимые ассоциации с ЯБ, рассмотрены их эпигенетические эффекты (использовались базы данных HaploReg (http://archive.broadinstitute.org/mammals/haploreg/haploreg.php) и GTExportal (http://www.gtexportal.org/) по ранее представленной методике [19-22].

\section{РЕЗУЛЬТАТЫ И ОБСУЖДЕНИЕ}

Данные по распределению частот генотипов и аллелей в исследуемых группах мужчин и женщин с ЯБ и контроля, а также в рассматриваемой выборке в целом представлены в табл. 1. По всем изученным десяти локусам генов $M M P$ наблюдаемое распределение генотипов соответствовало ожидаемому распределению согласно закону Харди-Вайнберга $\left(p_{\text {bonf }}>0.05\right)$.

Выявлены особенности ассоциации полиморфных локусов генов $M M P$ с развитием ЯБ у мужчин и женщин (табл. 2). Так, у мужчин с формированием заболевания были ассоциированы два полиморфных локуса гена $M M P 9$ - rs3918249, rs17576, тогда как у женщин с развитием заболевания связан полиморфный локус rs1799750 гена MMP1. При этом факторами риска возникновения ЯБ у мужчин были аллели $C$ rs 3918249 (coгласно доминантной модели ОШ $=1.61,95 \%$ ОШ $1.01-2.59, p=0.047, p_{\text {perm }}=0.048$ ) и $G \mathrm{rs} 17576$ (согласно аддитивной модели ОШ $=1.48,95 \%$ ОШ $1.06-2.07, p=0.023, p_{\text {perm }}=0.024$ и рецессивной модели ОШ $=2.08,95 \%$ ОШ 1.03-4.19, $p=0.040$, $\left.p_{\text {perm }}=0.042\right)$. У женщин аллель $2 G$ rs 1799750 гена $M M P 1$ в соответствии с аддитивной моделью имеет протективное значение при развитии заболевания (ОШ $=0.74,95 \%$ ОШ $0.57-0.96, p=0.021$, $\left.p_{\text {perm }}=0.047\right)$. Таким образом, согласно полученным нами данным полиморфизм rs3918249 и rs17576 гена $M M P 9$ определяет подверженность к развитию ЯБ у мужчин, a rs1799750 гена MMP1 у женщин.

Проведенный анализ эпигенетической роли полиморфных локусов, вовлеченных в формирование ЯБ у мужчин и женщин, показал следующее. Во-первых, локусы rs3918249 и rs 17576 гена MMP9 влияют на афинность мотивов ДНК к четырем и одному факторам транскрипции соответственно (Hmx, Hoxb8, Arid3a, Рax-5 и Рax-4 соответственно). Причем аллельные варианты этих локусов, ассоциированные с повышенным риском развития ЯБ у мужчин (аллели $C$ rs3918249 и $G$ rs 17576), определяют более высокое сродство к транскрипционным факторам Hmx ( $\Delta$ LOD scores для аллелей $C$ и $T \operatorname{rs} 3918249$ равен 1.9$)$, Нохb8 $(\Delta \mathrm{LOD}=2.9)$ и низкую афинность мотивов ДНК к Arid3a
$(\Delta \mathrm{LOD}=-0.7)$, Pax-5 $(\Delta \mathrm{LOD}=-3.9)$ и Рах-4 ( $\Delta$ LOD scores для аллелей $G$ и $A$ rs3918249 равен -2.1).

Во-вторых, полиморфный локус rs3918249 находится в регионе белков-гистонов, которые маркируют регуляторные области (промоторы, энхансеры) гена $M M P 9$ в слизистой оболочке желудка (H3K4me1_Enh) и двенадцитиперстной кишки (H3K4me3_Pro), гладкой мускулатуре желудка (H3K4me3_Pro) и двенадцатиперстной кишки (H3K4me1_Enh и H3K4me3_Pro) взрослого организма. Выраженный регуляторный потенциал имеет и rs17576, локализованный в области промоторных и энхансерных участков гена $M M P 9$ в различных отделах пищеварительной системы как плода (желудок - H3K4me1_Enh), так и взрослого организма: слизистая оболочка желудка (H3K4me1_Enh) и двенадцатиперстной кишки (H3K4me1_En̄h и H3K4me3_Рro), гладкая мускулатура желудка (H3K4me1_Enh и H3K4me3_Pro) и двенадцатиперстной кишки (H3K4me1_Enh и H3K4me3_Pro).

В-третьих, однонуклеотидный полиморфизм rs3918249 гена $M M P 9$ ассоциирован с экспрессией ряда генов (PLTP, NEURL2, RP3-337O18.9) в различных отделах пищеварительной системы: желудке $\left(P L T P, \beta=-0.25, p=1.7 \times 10^{-5}, p_{\mathrm{FDR}} \leq 0.05\right)$, пищеводе $\left(P L T P, \beta=-0.13, p=6.2 \times 10^{-5}, p_{\mathrm{FDR}} \leq\right.$ $\leq 0.05)$, толстом кишечнике $(P L T P, \beta=-0.25, p=$ $=1.7 \times 10^{-5}, p_{\mathrm{FDR}} \leq 0.05 ; N E U R L 2, \beta=-0.22, p=4.9 \times$ $\times 10^{-5}, p_{\mathrm{FDR}} \leq 0.05 ; R P 3-337018.9, \beta=-0.28, p=$ $\left.=3.5 \times 10^{-6}, p_{\mathrm{FDR}} \leq 0.05\right)$. Важное eQTL значение для разных отделов пищеварительной системы имеет и полиморфизм rs17576 гена $M M P 9-$ он связан с экспрессией трех генов PLTP, NEURL2, $R P 3-337018.9$ в пищеводе (PLTP, $\beta=-0.15, p=2.7 \times$ $\times 10^{-6}, p_{\mathrm{FDR}} \leq 0.05 ;$ NEURL $2, \beta=-0.25, p=1.8 \times$ $\times 10^{-5}, p_{\mathrm{FDR}} \leq 0.05 ; R P 3-337018.9, \beta=-0.23, p=$ $\left.=7.4 \times 10^{-5}, p_{\mathrm{FDR}} \leq 0.05\right)$ и толстом кишечнике (PLTP, $\beta=-0.18, p=5.6 \times 10^{-7}, p_{\mathrm{FDR}} \leq 0.05 ;$ NEURL2, $\beta=-0.23, p=1.3 \times 10^{-5}, p_{\mathrm{FDR}} \leq 0.05 ; R P 3-337018.9$, $\left.\beta=-0.28, p=1.9 \times 10^{-10}, p_{\mathrm{FDR}} \leq 0.05\right)$. При этом обращает на себя внимание факт того, что аллельные варианты этих локусов, ассоциированные с повышенным риском развития ЯБ у мужчин (аллели $C$ rs3918249 и $G$ rs17576), связаны с более низкой экспрессией $(\beta<0)$ всех трех вышерассмотренных генов (PLTP, NEURL2, RP3-337O18.9) в разных отделах пищеварительного тракта.

B-четвертых, выраженные эпигенетические эффекты характерны и для полиморфного локуса rs1799750 гена $M M P 1$, определяющего подверженность к развитию ЯБ у женщин: он влияет на сродство мотивов ДНК к 21 фактору транскрипции (AP-1, CHX10, DMRT2, Hoxb4, PLZF и др.), расположен в области модифицированных гистоновых белков, которые маркируют энхансеры 
Таблица 1. Частоты аллелей и генотипов полиморфных локусов генов $M M P$ в исследуемых группах мужчин и женщин с язвенной болезнью и в контроле

\begin{tabular}{|c|c|c|c|c|c|c|}
\hline \multirow[b]{2}{*}{ Локус } & \multirow{2}{*}{$\begin{array}{c}\text { Генотип, } \\
\text { минорный } \\
\text { аллель, } \\
\text { соответствие } \\
\text { НWE }\end{array}$} & \multicolumn{2}{|c|}{ Женщины $(n=441)$} & \multicolumn{2}{|c|}{ Мужчины $(n=305)$} & \multirow[b]{2}{*}{$\begin{array}{c}\text { Итого } \\
(n=746) \\
\%(n)\end{array}$} \\
\hline & & $\begin{array}{c}\text { контроль } \\
(n=230) \\
\%(n)\end{array}$ & $\begin{array}{c}\text { больные } \\
(n=211) \\
\%(n)\end{array}$ & $\begin{array}{c}\text { контроль } \\
(n=117) \\
\%(n)\end{array}$ & $\begin{array}{c}\text { больные } \\
(n=188) \\
\%(n)\end{array}$ & \\
\hline \multirow{5}{*}{$\begin{array}{l}\text { rs } 1940475 \\
M M P 8\end{array}$} & $C C$ & $26.52(61)$ & $32.23(68)$ & $30.17(35)$ & $25.00(46)$ & $28.34(210)$ \\
\hline & $C T$ & $45.65(105)$ & $41.71(88)$ & $45.69(53)$ & $49.46(91)$ & $45.48(337)$ \\
\hline & $T T$ & $27.83(64)$ & $26.06(55)$ & $24.14(28)$ & $25.54(47)$ & $26.18(194)$ \\
\hline & $T$ & 50.65 & 46.92 & 46.98 & 50.27 & 48.92 \\
\hline & $p_{\mathrm{HWE}}$ & 0.19 & 0.02 & 0.36 & 0.88 & 0.02 \\
\hline \multirow{5}{*}{$\begin{array}{l}\text { rs } 1799750 \\
M M P 1\end{array}$} & $1 G 1 G$ & $28.89(65)$ & $37.62(76)$ & $29.82(34)$ & $24.72(45)$ & $30.43(220)$ \\
\hline & $1 G 2 G$ & $44.44(100)$ & $43.56(88)$ & $48.25(55)$ & $50.56(92)$ & $46.33(335)$ \\
\hline & $2 G 2 G$ & $26.67(60)$ & $18.81(38)$ & $21.93(25)$ & $24.72(45)$ & $23.24(168)$ \\
\hline & $2 G$ & 48.89 & 40.59 & 46.05 & 50.00 & 46.40 \\
\hline & $p_{\mathrm{HWE}}$ & 0.11 & 0.19 & 0.85 & 1.00 & 0.07 \\
\hline \multirow{5}{*}{$\begin{array}{l}\text { rs679620 } \\
M M P 3\end{array}$} & $T T$ & $26.52(61)$ & $29.81(62)$ & $24.35(28)$ & $19.89(37)$ & $25.71(190)$ \\
\hline & $T C$ & $48.69(112)$ & $47.11(98)$ & $50.43(58)$ & $50.00(93)$ & $48.85(361)$ \\
\hline & $C C$ & $24.78(57)$ & $23.08(48)$ & $25.22(29)$ & $30.11(56)$ & $25.44(188)$ \\
\hline & $C$ & 49.13 & 46.63 & 50.43 & 55.11 & 49.86 \\
\hline & $p_{\mathrm{HWE}}$ & 0.69 & 0.46 & 1.00 & 1.00 & 0.55 \\
\hline \multirow{5}{*}{$\begin{array}{l}\text { rs243865 } \\
M M P 2\end{array}$} & $C C$ & $61.40(140)$ & 57.77 (119) & $48.69(56)$ & $55.92(104)$ & $57.24(419)$ \\
\hline & $C T$ & $31.58(72)$ & $35.44(73)$ & $44.35(51)$ & $34.95(65)$ & $35.66(261)$ \\
\hline & $T T$ & $7.02(16)$ & $6.79(14)$ & $6.96(8)$ & $7.53(14)$ & $7.10(52)$ \\
\hline & $T$ & 22.81 & 24.51 & 29.13 & 25.41 & 24.93 \\
\hline & $p_{\mathrm{HWE}}$ & 0.13 & 0.57 & 0.50 & 0.43 & 0.20 \\
\hline \multirow{5}{*}{$\begin{array}{l}\text { rs3918242 } \\
M M P 9\end{array}$} & $C C$ & $68.72(156)$ & $69.71(145)$ & $70.69(82)$ & $67.94(125)$ & $69.12(508)$ \\
\hline & $C T$ & $28.19(64)$ & $27.40(57)$ & $25.86(30)$ & $31.52(58)$ & $28.43(209)$ \\
\hline & $T T$ & $3.08(7)$ & $2.89(6)$ & $3.45(4)$ & $0.54(1)$ & $2.45(18)$ \\
\hline & $T$ & 17.18 & 16.59 & 16.38 & 16.30 & 16.67 \\
\hline & $p_{\mathrm{HWE}}$ & 0.82 & 0.81 & 0.50 & 0.06 & 0.59 \\
\hline \multirow{5}{*}{$\begin{array}{l}\text { rs3918249 } \\
\text { ММР9 }\end{array}$} & $T T$ & $37.83(87)$ & $32.68(67)$ & $47.83(55)$ & $36.22(67)$ & $37.55(276)$ \\
\hline & $T C$ & $43.48(100)$ & $53.66(110)$ & $40.00(46)$ & $48.65(90)$ & $47.07(346)$ \\
\hline & $C C$ & $18.69(43)$ & $13.66(28)$ & $12.17(14)$ & $15.13(28)$ & $15.38(113)$ \\
\hline & $C$ & 40.43 & 40.49 & 32.17 & 39.46 & 38.91 \\
\hline & $p_{\mathrm{HWE}}$ & 0.13 & 0.15 & 0.39 & 0.88 & 0.82 \\
\hline \multirow{5}{*}{ rs17576 MMP9 } & $A A$ & $37.83(87)$ & $34.60(73)$ & $47.41(55)$ & $37.10(69)$ & $38.22(284)$ \\
\hline & $A G$ & 47.39 (109) & $47.87(101)$ & $42.24(49)$ & $43.55(81)$ & $45.76(340)$ \\
\hline & $G G$ & $14.78(34)$ & $17.53(37)$ & $10.35(12)$ & $19.35(36)$ & 16.02 (119) \\
\hline & $G$ & 38.48 & 41.47 & 31.47 & 41.13 & 38.90 \\
\hline & $p_{\mathrm{HWE}}$ & 1.00 & 0.89 & 0.83 & 0.17 & 0.32 \\
\hline
\end{tabular}


Таблица 1. Окончание

\begin{tabular}{|c|c|c|c|c|c|c|}
\hline \multirow[b]{2}{*}{ Локус } & \multirow{2}{*}{$\begin{array}{c}\text { Генотип, } \\
\text { минорный } \\
\text { аллель, } \\
\text { соответствие } \\
\text { HWE }\end{array}$} & \multicolumn{2}{|c|}{ Женщины $(n=441)$} & \multicolumn{2}{|c|}{ Мужчины $(n=305)$} & \multirow[b]{2}{*}{$\begin{array}{c}\text { Итого } \\
(n=746) \\
\%(n)\end{array}$} \\
\hline & & $\begin{array}{c}\text { контроль } \\
(n=230) \\
\%(n)\end{array}$ & $\begin{array}{c}\text { больные } \\
(n=211) \\
\%(n)\end{array}$ & $\begin{array}{c}\text { контроль } \\
(n=117) \\
\%(n)\end{array}$ & $\begin{array}{c}\text { больные } \\
(n=188) \\
\%(n)\end{array}$ & \\
\hline \multirow{5}{*}{$\begin{array}{l}\text { rs } 3787268 \\
M M P 9\end{array}$} & $G G$ & $59.65(136)$ & $58.57(123)$ & $68.38(80)$ & 60.87 (112) & $61.03(451)$ \\
\hline & $G A$ & $35.96(82)$ & $37.62(79)$ & $28.20(33)$ & $34.78(64)$ & $34.91(258)$ \\
\hline & $A A$ & $4.39(10)$ & $3.81(8)$ & $3.42(4)$ & $4.35(8)$ & $4.06(30)$ \\
\hline & $A$ & 22.37 & 22.62 & 17.52 & 21.74 & 21.52 \\
\hline & $p_{\mathrm{HWE}}$ & 0.70 & 0.33 & 0.75 & 1.00 & 0.39 \\
\hline \multirow{5}{*}{$\begin{array}{l}\text { rs } 2250889 \\
M M P 9\end{array}$} & $C C$ & $76.55(173)$ & $80.86(169)$ & $81.03(94)$ & $83.16(153)$ & $80.14(589)$ \\
\hline & $C G$ & $20.80(47)$ & $16.75(35)$ & $16.38(19)$ & $16.30(30)$ & $17.82(131)$ \\
\hline & $G G$ & $2.65(6)$ & $2.39(5)$ & $2.59(3)$ & $0.54(1)$ & $2.04(15)$ \\
\hline & $G$ & 13.05 & 10.77 & 10.78 & 8.70 & 10.95 \\
\hline & $p_{\mathrm{HWE}}$ & 0.23 & 0.07 & 0.12 & 1.00 & 0.02 \\
\hline \multirow{5}{*}{ rs17577 MMP9 } & $G G$ & $68.75(154)$ & $69.42(143)$ & $70.69(82)$ & $66.67(120)$ & 68.73 (499) \\
\hline & $A G$ & $27.23(61)$ & $28.15(58)$ & $25.86(30)$ & $32.22(58)$ & $28.51(207)$ \\
\hline & $A A$ & $4.02(9)$ & $2.43(5)$ & $3.45(4)$ & $1.11(2)$ & $2.76(20)$ \\
\hline & $A$ & 17.63 & 16.50 & 16.38 & 17.22 & 17.01 \\
\hline & $p_{\mathrm{HWE}}$ & 0.36 & 1.00 & 0.50 & 0.11 & 0.89 \\
\hline
\end{tabular}

(H3K4me1_Enh) и “активные” энхансеры (Н3K27ac_Enh) в желудке и тонком кишечнике плода, слизистой оболочке желудка (Н3K4me1_Enh) и двенадцатиперстной кишки (Н3K4me1_Enh), гладкой мускулатуре желудка (Н3K27ac_Enh) и двенадцатиперстной кишки (Н3K27ac_Enh) взрослого организма, находится в регионе взаимодействия с двумя регуляторными белками - CFOS и GATA2. При этом следует отметить, что лишь для шести факторов транскрипции (AP-1, Dbx1, En-1, Evi-1, HMG-IY, Pou3f2) аллель $2 G$ rs1799750 гена $M M P 1$, связанный с низким риском развития ЯБ у женшин, повышает афинность ДНК ( $\Delta$ LOD $>0)$, тогда как для подавляющего числа транскрипционных факторов - 16 (CHX10, DMRT2, Hoxb4, PLZF, Pax-4, Pax-6 и др.) данный полиморфизм снижает сродство регуляторных мотивов ДНК $(\Delta \mathrm{LOD}<0)$.

В-пятых, с транскрипционной активностью гена $M M P 1$ в пищеварительной системе (пищевод) ассоциирован и rs1799750 гена MMP1 $(\beta=-0.30$, $\left.p=2.8 \times 10^{-7}, p_{\mathrm{FDR}} \leq 0.05\right)$. При этом аллель $2 G$ rs1799750, имеющий протективное значение при развитии ЯБ у женщин, связан с более высоким уровнем экспрессии гена $M M P 1$ (для референсного ему аллеля $1 G \beta<0$ ).

Итак, полученные нами данные указывают на выраженные эпигенетические эффекты поли- морфных локусов rs3918249 и rs 17576 гена $M М P 9$, и rs1799750 гена MMP1, которые они проявляют в различных отделах пищеварительной системы и в том числе органах-мишенях, поражаемых при язвенной болезни, - желудке и двенадцатиперстной кишке. Следует отметить, что полиморфные локусы rs3918249 и rs 17576 гена $M M P 9$ находятся на расстоянии 2.1 тыс. пн друг от друга и сильно сцеплены между собой $\left(r^{2}=0.99, D^{\prime}=0.99\right)$, поэтому их функциональные эффекты могут перекрываться.

Следует отметить, что в доступной нам литературе представлены лишь единичные работы, посвященные изучению роли полиморфизма генов $M M P$ в формировании язвенной болезни [13, 14, 23, 24], среди которых на модели российской популяции выполнено лишь одно исследование. Так, в работе Шаймардановой и др. [14] были изучены ассоциации с развитием ЯБ в популяции Республики Башкортостан трех из десяти изученных в нашей работе полиморфных локусов генов $M M P$ : rs1799750 гена $M M P 1$, rs3918242 и rs17576 гена $M M P 9$. Для двух из этих трех полиморфных локусов авторы установили ассоциации с заболеванием: генотип $1 G / 2 G$ rs1799750 гена $M M P 1$ являлся фактором риска развития ЯБ у татар $(\mathrm{OR}=1.94, p=0.02)$, а rs 17576 гена $M M P 9$ ассоциирован с развитием ЯБ у татар (для генотипа $A / A \mathrm{OR}=0.49, p=0.007$ и 


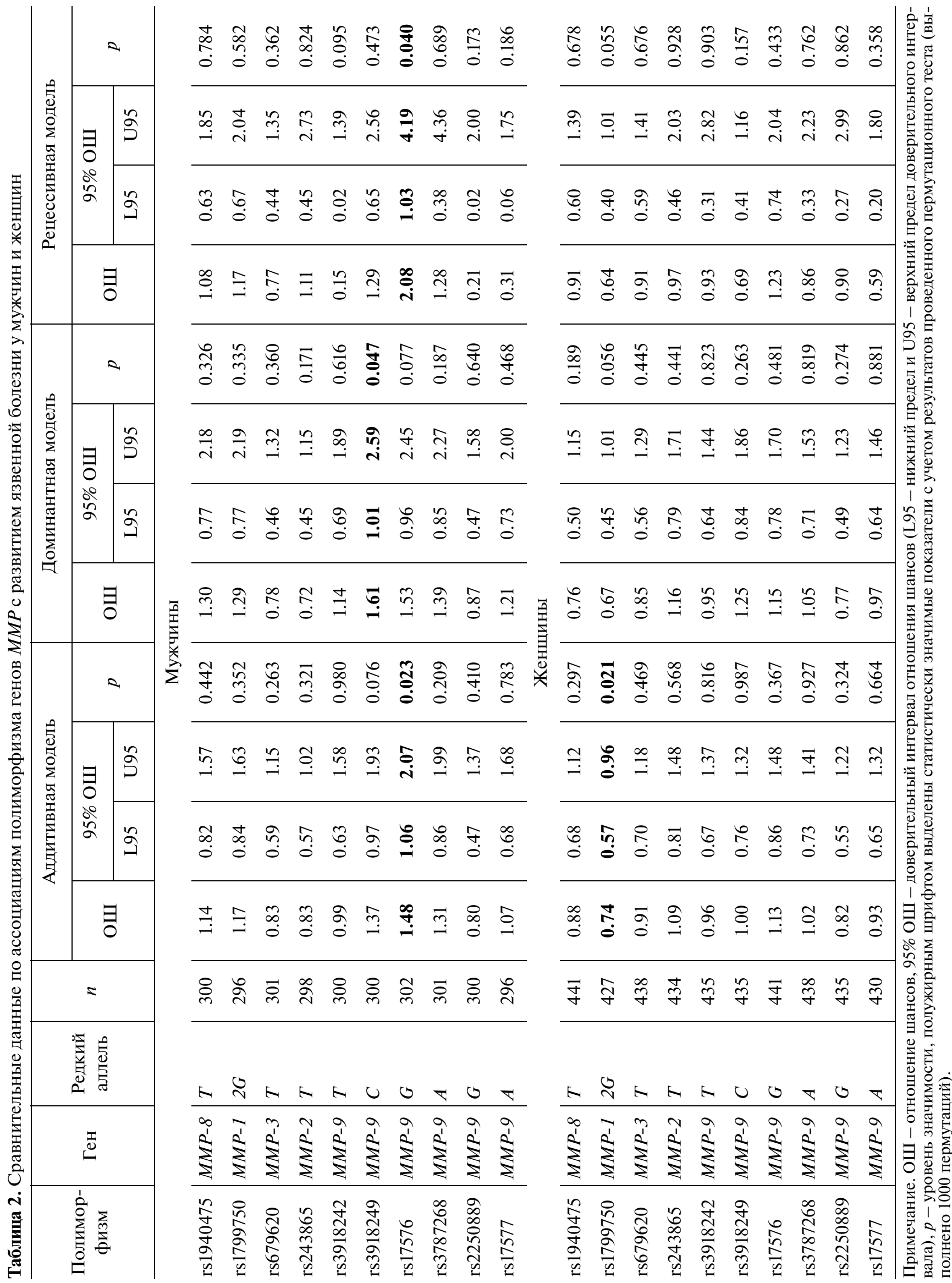


для генотипа $A / G \mathrm{OR}=2.19, p=0.003)$, формированием язвенной болезни двенадцатиперстной кишки (для генотипа $A / G \mathrm{OR}=1.57, p=0.009$ ) и развитием ЯБ у татар на фоне $H$. pylori инфекции (для генотипа $A / A \mathrm{OR}=0.54, p=0.03$ и для генотипа $A / G \mathrm{OR}=2.23, p=0.009)$.

Полученные нами данные о рисковом значении для развития ЯБ у мужчин аллеля $G$ rs 17576 и соответственно протективной роли в формировании заболевания референсного для него аллеля $A$ полностью согласуются с результатами работы Шаймардановой и др. [14], но отличаются от материалов, полученных в исследованиях Yeh et al. [13] и Hellmig et al. [23]. В работе Yeh et al. [13] не выявлено значимых ассоциаций rs17576 MMP9 с развитием $H$. pylori-позитивной язвенной болезни желудка и язвенной болезни двенадцатиперстной кишки в тайваньской популяции, а в исследовании Hellmig et al. [23], проведенном в популяции Германии, наоборот, обнаружено рисковое значение для возникновения $H$. pylori-позитивной язвенной болезни желудка аллеля $A$ rs 17576 . В работе Shan et al. [24], так же как и в нашем исследовании, не установлено статистически значимых связей rs3918242 гена MMP9 с формированием язвенной болезни двенадцатиперстной кишки у детей в китайской популяции.

Имеются многочисленные исследования, указывающие на связь рассматриваемых в настоящей работе полиморфных локусов генов $M M P 1$ и $M M P 9$ с онкологическими заболеваниями пищеварительной системы [25-33]. Наибольшее количество данных, подтверждающих вовлеченность в формирование злокачественных заболеваний пищеварительного тракта, получено для rs 1799750 гена MMP1: выявлена связь данного полиморфизма с раком пищевода (аденокарцинома пищевода) [25-28] и раком желудка [29-32]. В работе Okada et al. [33] показаны ассоциации rs17576 MMP9 с раком желудка как самостоятельно, так и в составе гаплотипа CAA rs3918242-rs17576-rs17577 MMP-9. Таким образом, можно сделать заключение о наличии общих генетических механизмов (в виде функционально значимых полиморфных локусов генов $M M P 1$ и $M M P 9)$, определяющих предрасположенность как к язвенной болезни, так и к онкологическим заболеваниям пищеварительной системы.

Установлены гендерные особенности ассоциаций полиморфизма генов $M M P$ с развитием ЯБ: rs3918249 и rs17576 гена ММР9 определяют подверженность к развитию ЯБ у мужчин, a rs1799750 гена $M M P 1-$ у женщин. Факторами риска возникновения ЯБ у мужчин являются аллели $C$ rs3918249 (ОШ $\left.=1.61, p_{\text {perm }}=0.048\right)$ и $G$ rs17576 $\left(\mathrm{OШ}=1.48-2.08, p_{\text {perm }} \leq 0.042\right)$ гена $M M P 9 . \mathrm{У}$ женщин протективное значение для развития заболевания имеет аллель $2 G$ rs 1799750 гена
MMP1 (ОШ $\left.=0.74, p_{\text {perm }}=0.047\right)$. Полиморфные локусы, ассоциированные с ЯБ у мужчин и женщин, проявляют выраженные эпигенетические эффекты (влияют на афинность мотивов ДНК к различным транскрипционным факторам, расположены в регионе промоторов и энхансеров в желудке и двенадцатиперстной кишке, связаны с экспрессией генов в различных отделах пищеварительной системы).

Все процедуры, выполненные в исследовании с участием людей, соответствуют этическим стандартам институционального и/или национального комитета по исследовательской этике и Хельсинкской декларации 1964 г. и ее последующим изменениям или сопоставимым нормам этики.

От каждого из включенных в исследование участников было получено информированное добровольное согласие.

Авторы заявляют, что у них нет конфликта интересов.

\section{СПИСОК ЛИТЕРАТУРЫ}

1. Milivojevic V., Milosavljevic T. Burden of gastroduodenal diseases from the global perspective // Curr. Treat. Options Gastro. 2020. V. 18. P. 148-157. https:// doi.org/10.1007/s11938-020-00277-z

2. Ramakrishnan K., Salinas R.C. Peptic ulcer disease // Am. Fam. Physician. 2007. V. 76. P. 1005-1012.

3. Здравоохранение в России. 2019: Стат. сб. М.: Росстат., 2019. $170 \mathrm{c}$.

4. Ивашкин В.Т., Маев И.В., Царьков П.В. и др. Диагностика и лечение язвенной болезни у взрослых (клинические рекомендации Российской гастроэнтерологической ассоциации, Российского общества колоректальных хирургов и Российского эндоскопического общества) // Рос. журн. гастроэнтерологии, гепатологии, колопроктологии. 2020. T. 30. № 1. C. 49-70. https://doi.org/ 10.22416/1382-4376-2020-30-1-49-70

5. Narayanan M., Reddy K.M., Marsicano E. Peptic ulcer disease and Helicobacter pylori infection // Missouri Med. 2018. V. 115(3). P. 219-224.

6. Lanas A., Chan F. Peptic ulcer disease // Lancet. 2017. V. 390(10094). P. 613-624. https://doi.org/ 10.1016/S0140-6736(16)32404-7

7. Saarialho-Kere U.K., Vaalamo M., Puolakkainen P. et al. Enhanced expression of matrilysin, collagenase, and stromelysin-1 in gastrointestinal ulcers // Am. J. Pathol. 1996. V. 148(2). P. 519-526.

8. Elkington P.T., O'Kane C.M., Friedland J.S. The paradox of matrix metalloproteinases in infectious disease // Clin. Exp. Immunol. 2005. V. 142(1). P. 12-20. https:// doi.org/10.1111/j.1365-2249.2005.02840.x

9. Tomita M., Ando T., Minami M. et al. Potential role for matrix metalloproteinase-3 in gastric ulcer healing // Digestion. 2009. V. 79(1). P. 23-29. https://doi.org/ $10.1159 / 000203637$ 
10. Kubben F.J., Sier C.F., Schram M.T. et al. Eradication of Helicobacter pylori infection favourably affects altered gastric mucosal MMP-9 levels // Helicobacter. 2007. V. 12(5). P. 498-504. https://doi.org/10.1111/j. 1523-5378.2007.00527.x

11. Li S.L., Zhao J.R., Ren X.Y. et al. Increased expression of matrix metalloproteinase- 9 associated with gastric ulcer recurrence // World J. Gastroenterol. 2013. V. 19(28). P. 4590-4595. https:// doi.org/10.3748/wjg.v19.i28.4590

12. Cheng H.C., Yang H.B., Chang W.L. et al. Expressions of MMPs and TIMP-1 in gastric ulcers may differentiate $H$. pylori-infected from NSAID-related ulcers // Sci. World J. 2012. V. 2012. P. 539316. https://doi.org/10.1100/2012/539316

13. Yeh Y.C., Cheng H.C., Chang W.L. et al. Matrix metalloproteinase-3 promoter polymorphisms but not dupA$H$. pylori correlate to duodenal ulcers in $H$. pyloriinfected females // BMC Microbiol. 2010. V. 10. P. 218. https://doi.org/10.1186/1471-2180-10-218

14. Шаймарданова Э.Х., Нургалиева А.Х., Хидиятова И.М. $u$ $\partial p$. Роль аллельных генов матриксных металлопротеиназ и их тканевых ингибиторов в развитии язвенной болезни // Генетика. 2016. Т. 52. № 3. C. 364-375. https://doi.org/10.7868/ S0016675816020119

15. Litovkina O., Nekipelova E., Dvornyk V. et al. Genes involved in the regulation of vascular homeostasis determine renal survival rate in patients with chronic glo-merulonephritis // Gene. 2014. V. 546(1). P. 112-116. https://doi.org/10.1016/j.gene. 2014.04.020

16. Reshetnikov E.A., Akulova L.Y., Dobrodomova I.S. et al. The insertion-deletion polymorphism of the ACE gene is associated with increased blood pressure in women at the end of pregnancy // J. Renin Angiotensin Aldosterone Syst. 2015. V. 16(3). P. 623-632. https://doi.org/ $10.1177 / 1470320313501217$

17. Миняйло О.Н. Распределение аллелей и гаплоблочная структура полиморфизма генов матриксных металлопротеиназ у больных $H$. pylori-негативной язвенной болезнью желудка и двенадцатиперстной кишки // Науч. результаты биомед. исследований. 2020. Т. 6. № 4. С. 488-502. https://doi.org/ 10.18413/2658-6533-2020-6-4-0-5

18. Moskalenko M., Ponomarenko I., Reshetnikov E. et al. Polymorphisms of the matrix metalloproteinase genes are associated with essential hypertension in a Caucasian population of Central Russia // Sci. Rep. 2021. V. 11(1). P. 5224. https://doi.org/10.1038/ s41598-021-84645-4

19. Ponomarenko I., Reshetnikov E., Polonikov A. et al. Candidate genes for age at menarche are associated with en-dometrial hyperplasia // Gene. 2020. V. 757. P. 144933. https://doi.org/10.1016/j.gene.2020.144933

20. Ponomarenko I., Reshetnikov E., Altuchova O. et al. Association of genetic polymorphisms with age at menarche in Russian women // Gene. 2019. V. 686. P. 228- 236. https://doi.org/10.1016/j.gene.2018.11.042

21. Ponomarenko I., Reshetnikov E., Polonikov A. et al. Candidate genes for age at menarche are associated with en-dometriosis // Reprod. Biomed. Online. 2020. V. 41(5).P. 943-956. https://doi.org/10.1016/j.rbmo. 2020.04 .016
22. Ponomarenko I., Reshetnikov E., Polonikov A. et al. Candidate genes for age at menarche are associated with uterine leiomyoma // Front. Genet. 2021. V. 11. P. 512940. https://doi.org/10.3389/fgene. 2020.512940

23. Hellmig S., Ott $S$., Rosenstiel P. et al. Genetic variants in matrix metalloproteinase genes are associated with development of gastric ulcer in $H$. pylori infection // Am.

J. Gastroenterol. 2006. V. 101. P. 29-35.

24. Shan Q.W, Jing C.X., Wang L.L. et al. Relationship between gene polymorphisms in MMP-9 and Helicobacter pylori-related upper gastrointestinal disease in children // Zhongguo Dang Dai Er Ke Za Zhi. 2010. V. 12(4). P. 262-266. Chinese.

25. Bradbury P.A., Zhai R., Hopkins J. et al. Matrix metalloproteinase 1, 3 and 12 polymorphisms and esophageal adenocarcinoma risk and prognosis // Carcinogenesis. 2009. V. 30(5). P. 793-798. https://doi.org/10.1093/ carcin/bgp065

26. Cheung W.Y., Zhai R., Bradbury P. et al. Single nucleotide polymorphisms in the matrix metalloproteinase gene family and the frequency and duration of gastroesophageal reflux disease influence the risk of esophageal adenocarcinoma // Int. J. Cancer. 2012. V. 131(11). P. 2478-2486. https://doi.org/ $10.1002 / \mathrm{ijc} .27541$

27. Guan X., Wang X., Luo H. et al. Matrix metalloproteinase 1, 3, and 9 polymorphisms and esophageal squamous cell carcinoma risk // Med. Sci. Monit. 2014.

V. 20. P. 2269-2274. https://doi.org/ 10.12659/MSM.892413

28. Tian J., Liu C., Liu G. et al. Cumulative evidence for association between genetic polymorphisms and esophageal cancer susceptibility: A review with evidence from meta-analysis and genome-wide association studies // Cancer Med. 2019. V. 8(3). P. 1289-1305. https://doi.org/10.1002/cam4.1972

29. Tian J., Liu G., Zuo C. et al. Genetic polymorphisms and gastric cancer risk: A comprehensive review synopsis from meta-analysis and genome-wide association studies // Cancer Biol. Med. 2019. V. 16(2). P. 361- 389. https://doi.org/10.20892/j.issn. 2095-3941.2018.0290

30. Yang M.D., Lin K.C., Lu M.C. et al. Contribution of matrix metalloproteinases-1 genotypes to gastric cancer susceptibility in Taiwan // Biomedicine (Taipei). 2017. V. 7(2). P. 10. https://doi.org/10.1051/ bmdcn/2017070203

31. Peng $Q ., X u Y$. Association between promoter polymorphisms of matrix metalloproteinase-1 and risk of gastric cancer // Onco. Targets Ther. 2015. V. 8. P. 2519-2526. https://doi.org/10.2147/OTT.S83004

32. Devulapalli K., Bhayal A.C., Porike S.K. Role of interstitial collagenase gene promoter polymorphism in the etiology of gastric cancer // Saudi J. Gastroenterol. 2014. V. 20(5). P. 309-314. https:// doi.org/10.4103/1319-3767.141693

33. Okada R., Naito M., Hattori Y. et al. Matrix metalloproteinase 9 gene polymorphisms are associated with a multiple family history of gastric cancer // Gastric Cancer. 2017. V. 20(2). P. 246-253. https://doi.org/ $10.1007 / \mathrm{s} 10120-016-0608-2$ 


\title{
Gender-Specific Features of Associations of Polymorphism of Matrix Metalloproteinase Genes with the Development of Peptic Ulcer Disease in the Population of the Central Chernozem Region of Russia
}

\author{
O. N. Minyaylo ${ }^{a}$, I. V. Ponomarenko ${ }^{a}$, and M. I. Churnosov ${ }^{a, ~ * ~}$ \\ ${ }^{a}$ Belgorod State University, Belgorod, 308015 Russia \\ *e-mail: churnosov@bsu.edu.ru
}

\begin{abstract}
Gender-specific features of associations of functionally significant polymorphic loci of matrix metalloproteinase genes with the development of peptic ulcer disease (PUD) in the population of the European part of Russia were studied. The study included 305 men (patients with PUD-188, control group-117) and 441 women (patients with PUD - 211, control group - 230). Ten functionally significant polymorphic loci of matrix metalloproteinase genes were genotyped: rs1799750 MMP1, rs243865 MMP2, rs679620 MMP3, rs1940475 $M M P 8$, rs3918242, rs3918249, rs17576, rs3787268, rs2250889, rs 17577 of the MMP9 gene. Associations of polymorphic loci of $M M P$ genes with PUD were studied by logistic regression (dominant, recessive and additive genetic models of allele interaction were evaluated). It was found that alleles $C$ rs3918249 (OR $=1.61$, $\left.p_{\text {perm }}=0.048\right)$ and $G$ rs $17576\left(\mathrm{OR}=1.48-2.08, p_{\text {perm }} \leq 0.042\right)$ of the $M M P 9$ gene were risk factors for the occurrence of PUD in men. In women, the $2 G$ rs1799750 allele of the MMP1 gene had a protective value for the development of the disease $\left(\mathrm{OR}=0.74, p_{\text {perm }}=0.047\right)$. Polymorphic loci associated with PUD in men and women exhibit pronounced epigenetic effects (they affect the affinity of DNA motifs to various transcription factors, are located in the region of promoters and enhancers in the stomach and duodenum, and are associated with gene expression in various parts of the digestive system). Gender-specific features of associations of polymorphism of $M M P$ genes with the development of PUD were revealed: rs3918249 and rs17576 of the $M M P 9$ gene determine the susceptibility to the development of PUD in men, and rs1799750 of the MMP1 gene - in women.
\end{abstract}

Keywords: peptic ulcer disease, matrix metalloproteinases, polymorphism, associations, gender. 